\title{
DIMENSIONAMENTO DE PAINÉIS POROSOS PARA CONDICIONAMENTO DO AR EM AMBIENTES CLIMATIZADOS
}

José Francisco Vilela Rosa ${ }^{1}$, Ilda de Fátima Ferreira Tinôco², Carlos Magno Fernandes ${ }^{3}$, Sergio Zolnier ${ }^{4}$

\section{RESUMO}

Painéis de resfriamento em celulose são comumente empregados no arrefecimento do ar, porém, alguns problemas estão associados a este material. Muitos estudos já concluíram que é viável o uso de materiais alternativos diversos em substituição a celulose, entre eles, aparece como viável, a argila. Em pesquisas anteriormente realizadas foi demonstrado que essa argila possui características favoráveis, que satisfazem as exigências pertinentes a um bom material poroso, podendo substituir, com vantagem econômica, a celulose. Diante do exposto, este trabalho teve como objetivo obter um dimensionamento de placas porosas em argila expandida, que permita, por meio de determinada granulometria da argila e uma espessura do painel, no qual se obtenha melhor eficiência de resfriamento do ar, o emprego em acondicionamento de ambientes em geral. Pelos resultados obtidos, pôde-se concluir que os painéis de argila expandida com granulometria 1 (referência à brita 1) e espessura de 7,7 cm foram os que possibilitaram melhor eficiência de resfriamento adiabático evaporativo para qualquer velocidade do ar compreendida entre 0,44 e 1,28 m/s.

Palavras-chave: argila expandida, painel evaporativo, eficiência de resfriamento e resfriamento adiabático evaporativo, granulometria.

\section{ABSTRACT \\ DIMENSIONING OF POROUS PADS OF EXPANDED CLAY FOR COOLING SYSTEMS OF ACCLIMATIZED ENVIRONMENTS}

Cellulose pad panels are commonly used in air cooling systems, despite some problems. Several studies have shown the possibility of using alternative materials such as expanded clay (Cinasita). Earlier studies have shown that this type of clay has required characteristics of a good porous material, thus can be an economical replacement for cellulose. The present study was done to determine suitable size and thickness of porous expanded clay pads, which could provide better cooling efficiency in acclimatized environments. Expanded clay pads with granulometry 1 (reference to grit 1) and $77 \mathrm{~mm}$ in thickness showed good efficiency for evaporative adiabatic cooling at the wind speed between 0.44 and $1.28 \mathrm{~m} / \mathrm{s}$.

Keywords: expanded clay, evaporative pads, cooling efficiency, evaporative adiabatic cooling, granulometry.

\author{
Recebido para publicação em 01/06/2009. Aprovado em 20/10/2011. \\ 1- Eng. Agrícola, Prof. Adjunto. D.S., Centro Univ. Newton Paiva, zevilela@terra.com.br \\ 2- Eng. Agrícola, Prof. Adjunta D.S., UFV - DEA - AMBIAGRO, iftinoco@ufv.br \\ 3- Prof. Adjunto. D.S. UFV - DEA, cmagno@ufv.br \\ 4- Eng. Agrícola Prof. Associado, D.S., UFV - DEA, zolnier@ufv.br
}

404 REVENG

404-411 p. ENGENHARIA NA AGRICULTURA, VIÇOSA - MG, V.20 N.5, SETEMBRO / OUTUBRO 2012 


\section{INTRODUÇÃO}

No agronegócio brasileiro, a produção animal é de grande importância, constituindo-se uma enorme fonte de divisas para o país. Em nível mundial, o Brasil talvez seja a nação que possua um dos maiores potenciais de crescimento de exportação. Entretanto, verifica-se que muitos animais não conseguem expressar todo o seu potencial produtivo sob as condições adversas do meio em que vivem. Assim, o ambiente constituise em um dos principais responsáveis pelo sucesso ou fracasso dos empreendimentos, podendo o mesmo ser definido como a soma dos impactos dos meios biológicos e físicos circundantes sobre os animais (CURTIS, 1983). No Brasil, os principais fatores limitantes de adaptação dos animais para a produção têm sido os altos valores de temperatura de bulbo seco, associados a altos valores de umidade relativa do ar nos ambientes de criação, que ocorrem em algumas regiões e especialmente no verão, o que pode reduzir a produtividade de algumas espécies (YANAGI JUNIOR, 2006).

Em uma de suas formas de climatização mais comuns e em expansão, a indústria de produção animal no Brasil vem buscando promover melhorias ao ambiente interno das instalações adotando sistemas de ventilação em modo túnel, associados aos sistemas de resfriamento evaporativo, composto de painéis de material poroso umedecido por gotejamento ou aspersor, através do qual o ar que o atravessa é resfriado antes de adentrar no alojamento. Usualmente são empregados painéis de celulose, que apresentam desempenho satisfatório no arrefecimento do ar, porém, os problemas associados a este material são: baixa durabilidade para as condições climáticas brasileiras, vulnerabilidade a ataque por roedores, dificuldade de aquisição (material patenteado importado) e custo elevado. Muitos estudos já concluíram que é viável o uso de material alternativo em substituição a celulose; entre eles aparece como viável, a argila expandida (cinasita), cujas principais vantagens são: leveza, resistência, estabilidade dimensional e incombustibilidade.

De acordo com Castro e Pimenta (2004), o resfriamento evaporativo é um processo natural que consiste na redução da temperatura do ar e elevação de sua umidade relativa através da incorporação de água ao ar e de mecanismos artificiais e/ou naturais que promovem simultânea transferência de calor e massa entre o ar e a água por ele incorporada. Tinôco e Resende (1997) relatam que nas atividades agrícolas esse processo tem sido empregado no arrefecimento de instalações. O processo consiste em mudanças do ponto psicrométrico de estado do ar, ou seja, à medida que se aumenta o conteúdo de água no ar, ocorre redução de sua temperatura, podendo ocorrer por aspersão, nebulização ou mesmo fazendo o ar atravessar uma parede porosa umedecida (placas evaporativas). Assim, o sistema de nebulização e o uso de placas evaporativas são duas diferentes maneiras de resfriamento evaporativo, que podem ser associadas à ventilação forçada de pressão positiva e/ou à ventilação de pressão negativa (TINÔCO \& RESENDE, 1997). Em pesquisa realizada em 2003 com equipamentos de resfriamento evaporativo, Camargo (2003) observou que esse sistema é mais eficiente quando as temperaturas são mais elevadas ou quando o ar apresenta baixa umidade relativa. Além disso, deve-se, sempre que psensação de resfriamento. $O$ consumo de energia de resfriadores evaporativos é muito inferior ao dos condicionadores de ar convencionais.

Sobre a espessura e a densidade de placas de material poroso, Wiersma e Stott (1983), Tinôco (1988) e Vigoderis (2002), afirmam que, à medida que se aumenta a espessura do material poroso, normalmente eleva-se a resistência à passagem do ar e, consequentemente, aumenta-se o tempo de contato do ar com o material poroso umedecido. Se para uma mesma espessura, for aumentada a densidade, a porosidade e a capilaridade do material poroso, a distribuição de água será uniforme, entretanto, torna-se necessário um maior volume de água para o umedecimento, aumentando desta maneira à resistência a ventilação do ar.

Tinôco et al. (2002), desenvolveram um estudo de comparação entre a eficiência de alguns materiais porosos alternativos encontrados no Brasil: cinasita (argila expandida), serragem, fibra vegetal e carvão, para o Sistema de Resfriamento Adiabático Evaporativo (SRAE). Nesse estudo foi possível concluir que a confecção de placas porosas para resfriadores evaporativos, utilizando- 
se os materiais cinasita e carvão vegetal, indica vantagens sobre os demais materiais estudados (serragem e fibra de madeira). No entanto, como não houve diferença estatística significativa $\mathrm{em}$ todos os horários, para os materiais testados e com base nos resultados de umidade relativa do ar e ITGU, sugeriu-se a condução de novas pesquisas, no sentido de se determinar a espessura correta da placa porosa.

Nesse sentido, este trabalho teve como objetivo obter um dimensionamento de placas porosas em argila expandida, que permita, por meio de determinada granulometria da argila e espessura do painel, alcançar melhor eficiência de resfriamento do ar para utilização em acondicionamento de ambientes em geral.

\section{MATERIAL E MÉTODOS}

O experimento foi realizado nos períodos de janeiro de 2007, abril de 2007 e janeiro de 2008, no Laboratório de Construções Rurais e Ambiência do Departamento de Engenharia Agrícola da Universidade Federal de Viçosa, localizado na

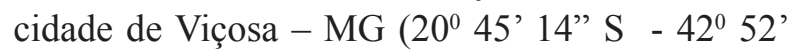
$55^{\prime \prime)}$, zona da mata e altitude $648 \mathrm{~m}$.

Foi utilizado nesta pesquisa um túnel de vento construído e desenvolvido por Vigoderis (2002), com base em protótipo de Liao e Chiu (2002), o qual permite testes de sistemas de resfriamentos evaporativos, com painéis evaporativos fabricados por materiais alternativos (Figura 1).

O material alternativo utilizado na pesquisa foi argila expandida com granulometria 1 (referência à brita 1) e granulometria 2 ( referência à brita 2 ).

O túnel de vento é composto pelas seguintes partes: câmara com 1,50 m de altura, $1,00 \mathrm{~m}$ de largura e 1,20 m de profundidade. Tais dimensões foram adotadas para que as características psicrométricas do ar pudessem ser avaliadas em no mínimo um metro de distância das placas porosas. O protótipo assim constituído possui uma abertura com dimensões de 1,23 m x 0,93 m, para o encaixe dos painéis porosos. Uma redução metálica foi fixada numa de suas extremidades, convergindo para um duto circular. Faz parte do sistema, um ventilador centrífugo de vazão de 1,6 $\mathrm{m}^{3} \mathrm{~s}^{-1}$, acionado por um motor de $5.500 \mathrm{~W}, 220 / 380$ Volts, trifásico, de $1750 \mathrm{rpm}$. A extremidade livre do ventilador possui um anteparo regulável com a função de variar a vazão do ar.

Durante os testes, os painéis de material poroso eram borrifados com água para permitir o molhamento da argila expandida, promovendo a evaporação e conseqüente resfriamento com a passagem do ar. O sistema de molhamento por lâmina de água foi constituído de um tubo de PVC, posicionado logo acima do painel, perfurado num total de 28 furos, por onde formavam jatos de água com uma lâmina homogênea que escoava

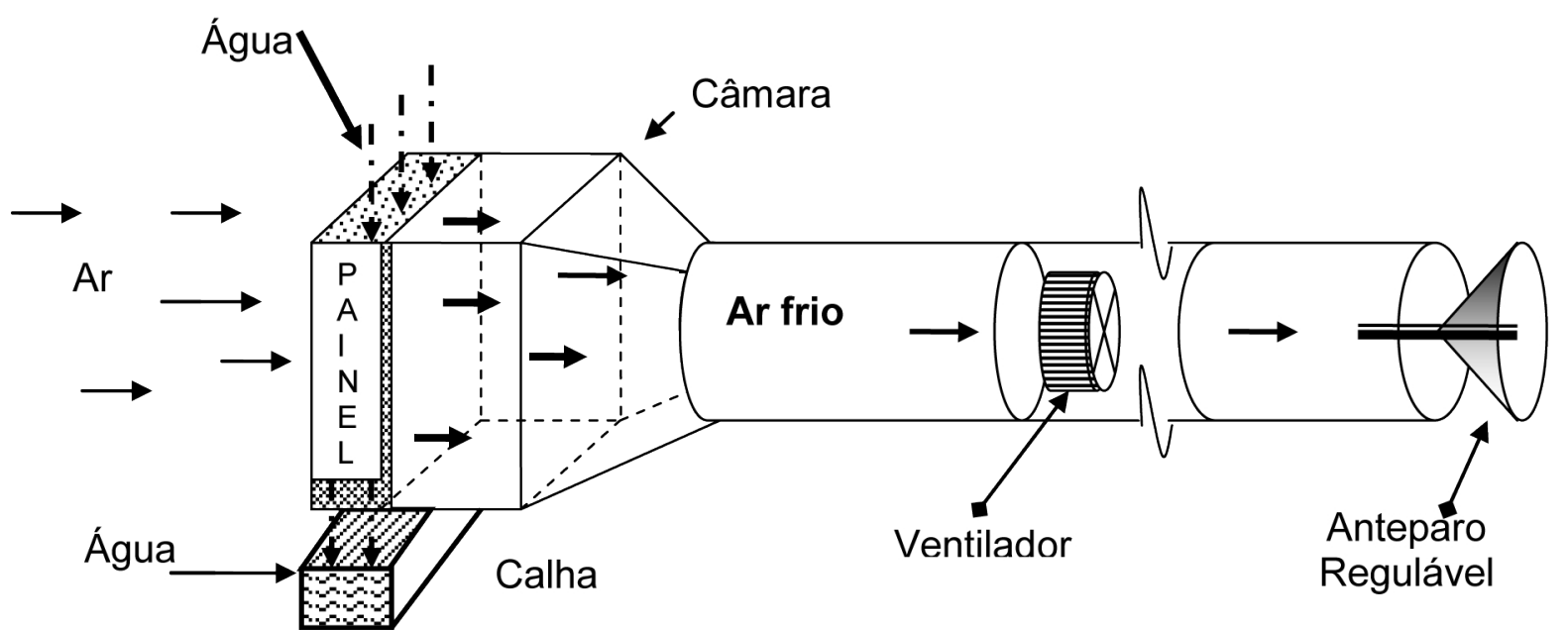

Fonte: autor.

Figura 1. Representação esquemática do túnel e do painel de resfriamento evaporativo. 
pelo material poroso. Este tubo era abastecido pela água de uma caixa d'água, e abaixo dos painéis evaporativos foi colocada uma calha metálica para a coleta da água excedente que deixava o material poroso.

Foram utilizados três painéis com dimensões fixas, com espessuras de 6,$0 ; 8,5 \mathrm{~cm}$ e $10,0 \mathrm{~cm}$, e, como testemunha, placas de celulose com proteção de tinta betuminosa na face externa. Foram medidos valores de temperatura de bulbo seco do ar externo e do ar interior, umidade relativa do ar externo, velocidade do ar próximo aos painéis evaporativos e pressão estática para quatro diferentes aberturas do regulador de vazão, em intervalos de 30 segundos.

A temperatura e a umidade relativa do ar foram medidas por meio de um instrumento que combina dois sensores independentes, sendo um para temperatura e outro para umidade relativa do ar (Modelo: HUM50Y, VAISALA, WOBURN, MA). Além desses sensores, termopares do tipo "T" foram instalados no interior do túnel para leituras das temperaturas. As velocidades do ar foram medidas posicionando o aparelho medidor no centro geométrico de 20 pontos, determinando-se a velocidade média do ar por cada repetição, antes de sua passagem pela placa porosa. Para essas leituras foi utilizado um anemômetro de fio quente (modelo: FMA-903-I, OMEGA, STANFORD). As leituras de pressão estática foram obtidas utilizando-se um Micro Manômetro do fabricante KIMO, modelo MP 120, com medidas em mm $\mathrm{H}_{2} 0$ e exatidão de \pm $2 \% \mathrm{~mm} \mathrm{H}_{2} 0$.

Os dados relativos ao ambiente externo próximo ao painel e no interior do túnel foram obtidos por meio de um sistema automático de aquisição de dados, tendo como plataforma um microcomputador. Nessa configuração, uma placa de aquisição de dados (CYDAS 1620HR, CYBERRESEARCH, BRANFORD, CT), de 16 canais de entrada analógica, com 16 bits, foi instalada em um barramento ISA do microcomputador. Além da placa principal, foi utilizada uma placa de expansão CYEXP32, para amplificação dos sinais provenientes dos termopares tipo T. Os canais analógicos foram conectados aos sensores responsáveis pelo monitoramento do ambiente físico.

Para a realização dos testes experimentais fezse a interação entre três fatores influenciadores no processo de resfriamento adiabático evaporativo: tipo de argila expandida, espessura do painel e vazão do ar.

Foram determinadas três espessuras de painéis para os testes: $6 \mathrm{~cm}\left(\mathrm{E}_{1}\right), 8,5 \mathrm{~cm}\left(\mathrm{E}_{2}\right)$ e $10 \mathrm{~cm}\left(\mathrm{E}_{3}\right)$. A cada abertura do regulador de vazão eram obtidas diferentes velocidades do ar, que variavam entre 0,44 e $1,28 \mathrm{~m} \mathrm{~s}^{-1}$, ficando denominadas de $\mathrm{V}_{1}, \mathrm{~V}_{2}$, $\mathrm{V}_{3}$ e $\mathrm{V}_{4}$, sendo; $\mathrm{V}_{1}$ a menor e $\mathrm{V}_{4}$ a maior velocidade de ar. Dessa forma, com duas granulometrias, três espessuras e quatro vazões, formaram-se 24 conjuntos de medidas para os testes; e, para cada conjunto foram feitas três séries de repetições.

A eficiência do resfriamento adiabático evaporativo $(\varepsilon)$ foi calculada por meio da equação (CASTRO E PIMENTA, 2004):

$\varepsilon=\frac{\mathrm{T}_{\text {ext }}-\mathrm{T}_{\text {int }}}{\mathrm{T}_{\mathrm{ext}}-\mathrm{T}_{\mathrm{B}}} 100 \%$

em que

$\mathrm{T}_{\text {ext }}=$ temperatura de bulbo seco do ar, exterior $\left({ }^{\circ} \mathrm{C}\right)$;

$\mathrm{T}_{\text {int }}=$ temperatura de bulbo seco do ar, interior $\left({ }^{\circ} \mathrm{C}\right)$; $\mathrm{T}_{\mathrm{BU}}=$ temperatura de bulbo molhado do ar, antes de ultrapassar o painel $\left({ }^{\circ} \mathrm{C}\right)$.

A determinação da temperatura de bulbo $\left(\mathrm{T}_{\mathrm{BU}}\right)$ molhado foi obtida por meio do método numérico de Newton, utilizando os valores psicrométricos medidos.

Para obter modelos estatísticos fez-se a relação entre todos os fatores entre si, obtendo equações ajustadas. Os dados foram analisados utilizando-se a análise de regressão linear múltipla. Foram consideradas como dependentes, as seguintes variáveis: Temperatura do ar externa (TE), Temperatura do ar de bulbo úmido (TU) e Temperatura do ar interna (TI). E, como independentes foram consideradas as seguintes variáveis: Espessura do painel (E) e Velocidade do ar externo (V).

Os modelos utilizados foram pré-definidos em função dos fatores influenciadores. Os parâmetros adotados para obtenção das equações foram definidos em função dos dados coletados da seguinte forma: 
granulometrias 1 e 2; espessuras dos painéis: $6,0 \mathrm{~cm} ; 8,5 \mathrm{~cm}$ e $10,0 \mathrm{~cm}$; para as velocidades determinou-se os valores médios para cada abertura do regulador de vazão ficando estipuladas para a granulometria 1 os valores; $0,44,0,72,1,00$ e $1,28 \mathrm{~m} \mathrm{~s}^{-1}$ e para a granulometria 2 os valores; $0,40,0,83,1,26$ e $1,70 \mathrm{~m} \mathrm{~s}^{-1}$.

Os modelos foram escolhidos fundamentados na significância da regressão utilizando-se o teste "t" de Student, adotando-se o nível de 1 e $5 \%$ de probabilidade no coeficiente de determinação. Para a análise estatística e obtenção dos modelos estatísticos dos resultados, utilizou-se o programa SAEG (Sistemas de Análises Estatísticas e Genéticas), versão 8.0, desenvolvido pela Universidade Federal de Viçosa.

\section{RESULTADOS}

Apresenta-se, por meio da Quadro 1, as equações ajustadas através de regressão linear múltiplas das variáveis dependentes TE (Temperatura do ar externo), TU (Temperatura de bulbo molhado) e TI (temperatura interna) em função da espessura (E) e da velocidade $(V)$ para as granulometrias 1 e 2 , acompanhadas por seus respectivos coeficientes de determinação.

Observa-se, no Quadro 1, que as equações ajustadas para os painéis com argila expandida de granulometria 2, são constantes e verifica-se que não houve efeito da espessura e da velocidade do ar para todas as características citadas, isto é, cada variável dependente analisada não sofreu influência nas faixas de variação de espessura ou de variação da velocidade. Para os painéis com argila expandida em granulometria 1, foi possível a construção de gráficos de cada variável dependente em função da espessura e da velocidade, dimensionando o painel para o seu melhor emprego. Os gráficos 1, 2 e 3 foram construídos a partir destas equações ajustadas. Apenas as argilas expandidas com granulometria 1 proporcionaram equações ajustadas possíveis de obter algum resultado, pois, argilas com granulometria 2 mostraram-se insuficientes para determinar a espessura ideal de um painel, na faixa das espessuras analisadas neste experimento, com esse material alternativo.

Por meio das equações ajustadas apresentadas na Tabela 1 e dos gráficos das figuras 1,2 e 3, determinou-se a espessura ideal dos painéis de argila expandida, esse valor é de aproximadamente 7,70 centímetros, onde se tem o ponto máximo do gráfico, mostrando que a eficiência atinge maior valor com a granulometria 1. Após esse valor, a eficiência começa a diminuir. De acordo com Vigoderis (2002), esse fenômeno pode ser explicado pela interferência da entrada de água na câmara do túnel, intensificado a partir deste ponto, e assim diminuindo a quantidade de água disponível para o processo de resfriamento adiabático evaporativo.

Quadro 1. Equações de regressão ajustadas das variáveis, TE (Temperatura do ar externo, em $\left.{ }^{\circ} \mathrm{C}\right)$, TU (Temperatura de bulbo molhado, em ${ }^{\circ} \mathrm{C}$ ) e TI (temperatura interna, em ${ }^{\circ} \mathrm{C}$ ) em função da espessura $\mathrm{E}$ e velocidade $\mathrm{V}$ para granulometrias 1 e 2, e seus respectivos coeficientes de determinação

\begin{tabular}{|c|c|c|c|}
\hline Gran & Var & Equações Ajustadas & $\mathbf{R}^{2}$ \\
\hline 1 & $\mathrm{TI}$ & $\mathrm{T} \hat{\imath}=-2,736+7,088 * * \mathrm{E}-0,469 * * \mathrm{E}^{2}+0,531 * \mathrm{~V}-0,129 * \mathrm{~V}^{2}$ & 0,97 \\
\hline 1 & TE & $\mathrm{Tê}=-0,58+7,157 * * \mathrm{E}-0,465^{* *} \mathrm{E}^{2}+0,477 * * \mathrm{~V}$ & 0,81 \\
\hline 1 & TU & $\mathrm{T} \hat{\mathrm{u}}=4,657+3,958 * * \mathrm{E}-0,252 * * \mathrm{E}^{2}+1,981 * * \mathrm{~V}-0,790 * * \mathrm{~V}^{2}$ & 0,89 \\
\hline 2 & TE & Tê $=26,98$ & - \\
\hline 2 & $\mathrm{Tu}$ & Tû $=20,58$ & - \\
\hline 2 & $\mathrm{TI}$ & $\mathrm{T} \hat{1}=22,52$ & - \\
\hline
\end{tabular}

** - Significativo em nível de $1 \%$ de probabilidade pelo teste " $t$ ".

- Significativo em nível de $5 \%$ de probabilidade pelo teste " $t$ ". 


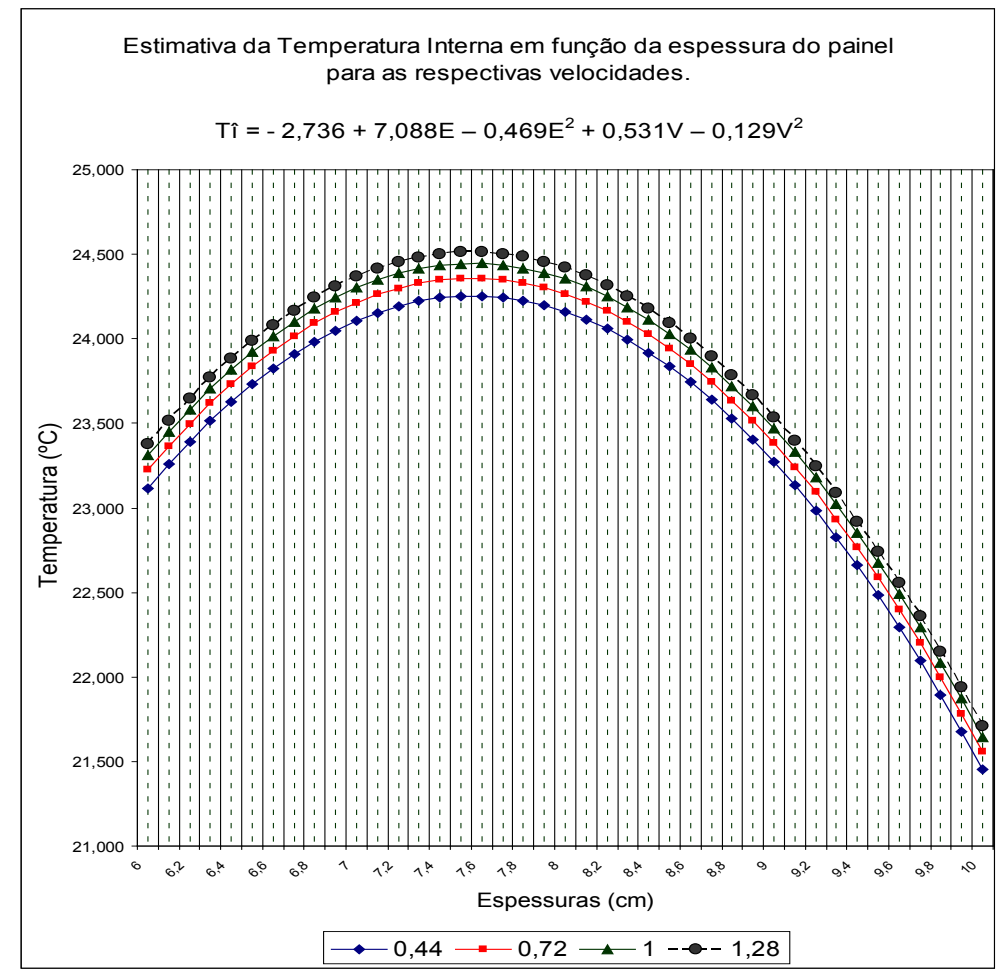

Figura 2. Gráfico da equação $T \hat{1}=-2,736+7,088 \mathrm{E}-0,469 \mathrm{E}^{2}+0,531 \mathrm{~V}-0,129 \mathrm{~V}^{2}$, variação da temperatura interna $\left({ }^{\circ} \mathrm{C}\right)$ em função da espessura do painel com argila expandida $(\mathrm{G} 1)$ para cada velocidade do ar adotada no experimento.

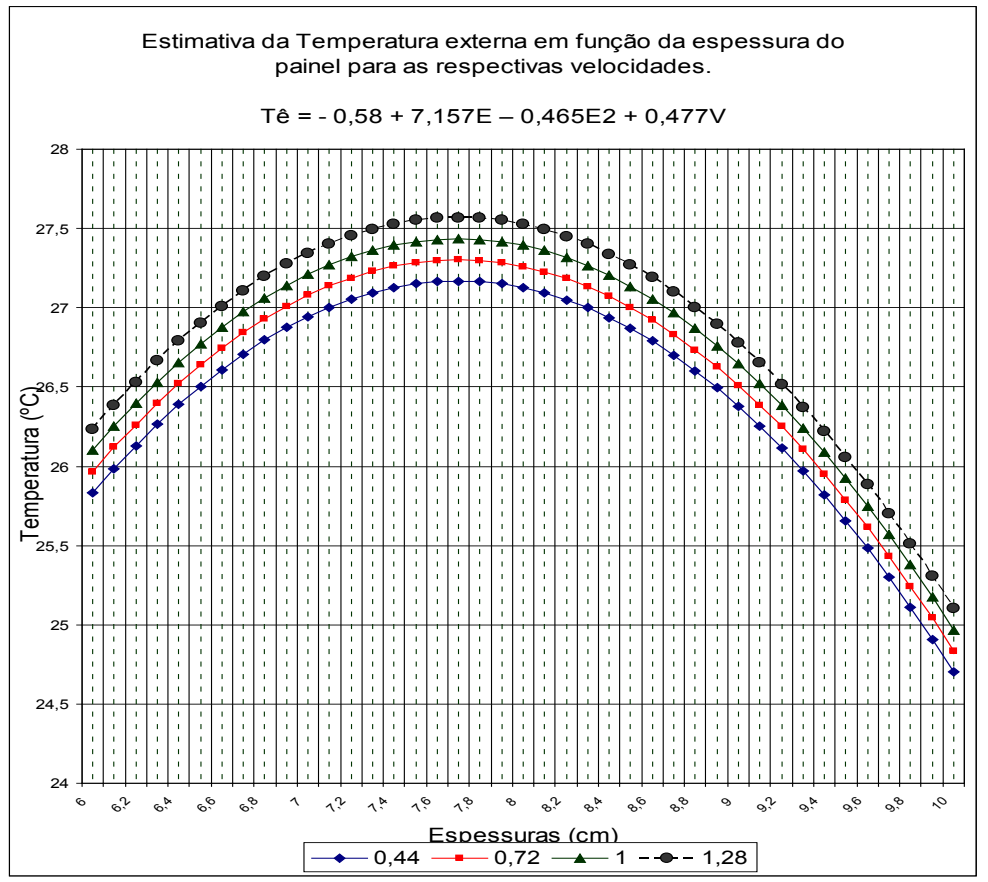

Figura 3. Gráfico da equação Tê $=-0,58+7,157 \mathrm{E}-0,465 \mathrm{E}^{2}+0,477 \mathrm{~V}$, variação da temperatura externa $\left({ }^{\circ} \mathrm{C}\right)$ em função da espessura do painel com argila expandida (G1) para cada velocidade do ar adotada no experimento. 


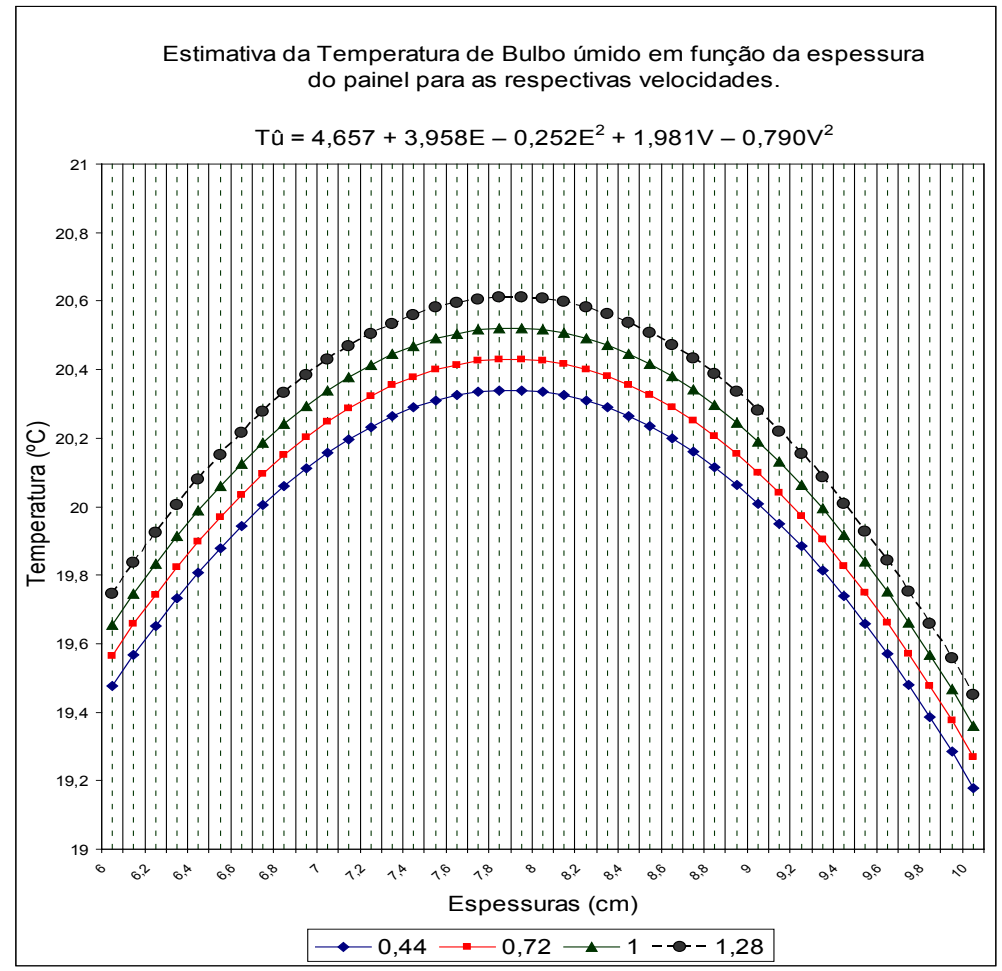

Figura 4. Gráfico da equação Tû $=4,657+3,958 \mathrm{E}-0,252 \mathrm{E}^{2}+0,1981 \mathrm{~V}-0,790 \mathrm{~V}^{2}$, variação da temperatura de bulbo molhado $\left({ }^{\circ} \mathrm{C}\right)$ em função da espessura do painel com argila expandida G1 para cada velocidade do ar adotada no experimento.Ox movilis pultio es tarestraet; nite tatiesces hoculiis, quam iam.

\section{CONCLUSÕES}

Pode-se concluir com este trabalho que:

- Foram encontrados melhores valores de eficiência de resfriamento do ar para os painéis evaporativos confeccionados com argila expandida na granulometria 1 (referência a brita 1) em relação a granulometria 2 (referência a brita 2), para todas as espessuras de painéis adotadas $(6,0 ; 8,5$ e $10,0 \mathrm{~cm})$;

- Os painéis de argila expandida com granulometria 1 e espessura de $7,7 \mathrm{~cm}$ foram identificados nos cálculos como sendo os que possibilitaram melhor eficiência de resfriamento adiabático evaporativo para qualquer velocidade do ar compreendida entre

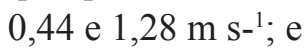

- Os modelos de eficiência de resfriamento, estimados por meio das médias das velocidades e das espessuras dos painéis, são apresentados pelas equações:

- $\mathrm{Ti}=-2,736+7,088 \mathrm{E}-0,469 \mathrm{E}^{2}+0,531 \mathrm{~V}-$ $0,129 \mathrm{~V}^{2}, \quad$ (1) $\quad \mathrm{r}^{2}=0,97$;

- $\mathrm{Ti}=-0,58+7,157 \mathrm{E}-0,465 \mathrm{E}^{2}+0,477 \mathrm{~V}$, (2) $r^{2}=0,81$;

- $\mathrm{Tu}=4,657+3,958 \mathrm{E}-0,252 \mathrm{E}^{2}+0,1981 \mathrm{~V}-$ $0,790 \mathrm{~V}^{2}, \quad$ (3) $\quad r^{2}=0,89$.

\section{REFERÊNCIAS BIBLIOGRÁFICAS}

CAMARGO, J.R. Sistemas de resfriamento evaporativo e Evaporativo-adsortivo aplicados ao Condicionamento de ar. 2003. 140f. Tese (Doutorado em Engenharia Mecânica) Guaratinguetá-SP: Engenharia do Campus de Guaratinguetá, Universidade Estadual Paulista, 2003.

CASTRO, W.P.; PIMENTA, J.M.D. Modelagem e simulação de painéis evaporativos diretos. Congresso Brasileiro de Engenharia e Ciências

\section{REVENG 404-411 p.}


Térmicas. 10. Anais... Rio de Janeiro, RJ, 2004.

CURTIS, S.E. Environmental management in animal agriculture. Ames: The Iowa State University Press, 1983. 409p.

LIAO, C.M; CHIU, K.H. Wind Tunnel Modeling the System Performance of Alternative Evaporative Cooling Pads in Taiwan Region. Building and Environment, v.37, p.177-187, 2002.

TINÔCO, I.F.F.; FIGUEIREDO, J.L.A.; Santos, R.C.; Paula, M.O.; Pugllesi, N.L.; Vigoderis. R, B. Avaliação de materiais alternativos utilizados na confecção de placas porosas para sistemas de resfriamento adiabático evaporativo. Revista Brasileira de Engenharia Agrícola e Ambiental, Campina Grande, v.6, n.1, p.147-150, 2002.

TINÔCO, I.F.F. Resfriamento adiabático (Evaporativo) na produção de frangos de corte. Viçosa - MG: UFV, 1988. 92 pag. Dissertação (Mestrado em construção rurais e ambiência Engenharia Agrícola). Nome da Instituição do programa de mestrado, Universidade Federal de Viçosa 1998.
TINÔCO, I.F.F; RESENDE,P.L. Produção de frango de corte em alta densidade. Manual CPT - Centro de Produções Técnicas. 20 pag., 1997.

VIGODERIS, R.B.; TINÔCO, I.F.F.; LACERDA FILHO, A.F.; SILVA, J.N., GATES, R.S., PAULI, D.G.; SILVA, C.E. \& GUIMARÃES, M.C.C. Construção de túnel de vento reduzido, visando a avaliação de argila expandida em sistemas de resfriamento adiabático evaporativo para arrefecimento térmico em galpões de produção animal. Engenharia na Agricultura, Viçosa, $\mathrm{MG}$, v.15, n.3, p.191-199, 2007.

WIERSMA, F.; STOTT, F.M. Response of dairy cattle to an evaporative cooled environment. Proceedings of the Livestock Environment. St. Joseph: American Society of Agricultural Engineers. 1974. p.88-95.

YANAGI JUNIOR, T. Inovações tecnológicas na bioclimatologia animal visando aumento da produção animal: relação bem estar animal $x$ clima. 2006. Artigo em hypertexto. Disponível em: $<$ http://www.infobibos.com/Artigos/2006_2/>. 